\title{
Surface relaxation and chemical exchange in hydrating cement pastes: A two-dimensional NMR relaxation study
}

\author{
P. J. McDonald, ${ }^{1}$ J.-P. Korb, ${ }^{2}$ J. Mitchell, ${ }^{1}$ and L. Monteilhet ${ }^{1,2}$ \\ ${ }^{1}$ Department of Physics, School of Electronics and Physical Sciences, University of Surrey, Guildford, GU2 7XH, United Kingdom \\ ${ }^{2}$ Laboratoire de Physique de la Matière Condensée, UMR 7643 CNRS, Ecole Polytechnique, 91128 Palaiseau, France
}

(Received 3 April 2005; revised manuscript received 20 May 2005; published 27 July 2005)

\begin{abstract}
We report the first nuclear magnetic resonance (NMR) two-dimensional correlation $T_{1}-T_{2}$ and $T_{2}-T_{2}$ measurements of hydrating cement pastes. A small but distinct cross peak in the two-dimensional relaxation spectrum provides the first direct evidence of chemical exchange of water between gel and capillary pores occurring over the first 14 days of hydration. A correlation of features along the line $T_{1}=4 T_{2}$ provides strong supportive evidence for the surface diffusion model of ${ }^{1} \mathrm{H}$ nuclear spin relaxation in cements and for a multimodal discrete pore size distribution. Differences in detail of the results are reported for white cement paste and white cement paste with added silica fume. Both the method and the theory presented can be applied more widely to other high surface area materials with other reactive surface areas.
\end{abstract}

DOI: 10.1103/PhysRevE.72.011409

PACS number(s): 82.70.-y, 81.05.Rm, 68.08.-p, 78.30.Ly

\section{INTRODUCTION}

The nuclear spin relaxation times of hydrogen nuclei within water molecules are determined by the detailed dynamics and chemical and physical environment of the water. In consequence, the measurement of ${ }^{1} \mathrm{H}$ nuclear spin relaxation times provides information on pore-water interactions and water dynamics in cements. Two relaxation times are generally measured in these ${ }^{1} \mathrm{H}$ NMR experiments: $T_{1}$, the spin-lattice relaxation time, and $T_{2}$, the spin-spin relaxation time. The $T_{1}$ is associated with dissipation processes from the spin system through all the other degrees of freedom (lattice). The $T_{2}$ controls the dephasing within the spin system. These times differ widely for chemically bound hydrogen, hydrogen adsorbed onto surfaces, and hydrogen in water confined to small pores. Additionally, the times are strongly influenced by the diffusion of water through large internal magnetic field gradients arising from discontinuities in magnetic susceptibilities at pore wall interfaces. The interpretation of relaxation measurements is further complicated by paramagnetic species present to some degree in every type of cement.

The standard model of relaxation in porous media is developed from original work by Brownstein and Tarr [1]. Hydrogen protons diffusing in the pore water occasionally encounter a pore surface. At the pore surface they are able to undergo relaxation. If the rate of diffusion across the pore is fast compared to the surface relaxivity, then a single averaged relaxation rate is observed proportional to the pore surface to volume ratio. If the diffusion rate is slower, and the surface relaxivity is very high, then separate modes are observed. In general, for a 3D bulk liquid, the models lead to the prediction that $T_{1}=T_{2}$.

There are already several studies to be found in the literature that report ${ }^{1} \mathrm{H}$ measurements of $T_{1}$ and $T_{2}$ relaxation during the hydration of cements. Multiple relaxation modes are normally found. Schreiner et al. [2] discussed a simple 3 -phase model that is been widely adapted to interpret results. The three phases are bound water, gel water and cap- illary water. Bound water, i.e., water that has chemically combined in calcium silicate hydrate (CSH) gel, has almost no mobility and exhibits long ${ }^{1} \mathrm{H} T_{1} \quad(>100 \mathrm{~ms})$ and extremely short $T_{2} \quad(\sim 10 \mu \mathrm{s})$ relaxation times. Water in the gel pores is more mobile but the relaxation is heavily dominated by surface interactions, and so both $T_{1}$ and $T_{2}$ are short $(0.5-1.0 \mathrm{~ms})$. The relaxation times of ${ }^{1} \mathrm{H}$ in water in the larger capillary pores are longer but still limited to approximately $5-10 \mathrm{~ms}$, much less than the bulk liquid times $(>1 \mathrm{~s})$. Schreiner et al. attributed this to the water being saturated with paramagnetic iron oxides. However, it is now more widely accepted that the capillary water relaxation times are also dominated by surface interactions. Consequently, the relaxation times depend on the surface-tovolume ratio of the pores and can therefore reveal the pore size. More complicated 5-phase [3] and 7-phase [4] models that incorporate surface layers of water in the pore structure have also been proposed.

Recently Korb and co-workers [5,6] considered more carefully the mechanism of the $T_{1}$ surface relaxivity. They proposed that motion of a water molecule across the surface consists of a two-dimensional random walk between binding sites characterized by a hopping time $\tau_{m}$. The water stays in the vicinity of the surface for a characteristic time $\tau_{s}$ after which it leaves the surface and reenters the pore liquid only to be replaced by another. Necessarily for the model, $\tau_{s}$ $\gg \tau_{m}$. While the water is near the surface, it undergoes repeated encounters with paramagnetic ions. The resultant modulation of the local dipolar magnetic field created by the paramagnetic ions and experienced by the hydrogen nuclei is the primary source of spin relaxation. It is found that the relaxation is strongly frequency dependent at low frequencies. The strong frequency dependence arises from the very different probability of a return to the origin for a random walk in two dimensions compared to a walk in three dimensions. Korb et al. tested the model by measuring the very low frequency dependence (down to $10 \mathrm{kHz}$ ) of the spin lattice relaxation rate $\left(R_{1}=1 / T_{1}\right)$ using field cycling methods [7].

In this work, we use the much easier methodology of two-dimensional (2D) $T_{1}-T_{2}$ correlation experiments to fur- 
ther test the model and to provide us with additional information on the hydrogen dynamics. These methods are afforded to us through the recent establishment by Venkataramanan and co-workers [8,9] of a robust means of making a two-dimensional inverse Laplace transform; a notoriously ill-posed problem. These correlation experiments, which can couple nuclear spin relaxation occurring during one time period with relaxation or self-diffusion occurring in a subsequent time period have previously been applied to oil-bearing rocks [10-12], complex fluids [13,14], and foodstuffs [15]. In oil bearing rocks they are used for the most part as an improved means of differentiating between oil and water. In other systems they are used for detailed analysis of microstructure.

To the best of our knowledge, this is the first time that $2 \mathrm{D}$ relaxation correlation methods have been applied to cement. As well as providing further evidence for the verity of the surface diffusion mechanism of relaxation in cements, the experiments yield the first strong evidence of chemical exchange of hydrogen between pores of different size, critically between gel pores and capillary pores. The former, although not chemically bound into the solid structure of the cement, is nonetheless an integral part of the CSH. Its removal is generally associated with damage to the delicate CSH structure. The latter is readily evaporable water in the pore structure created by shrinkage of the CSH during chemical cure and hydration. The objective of much advanced cementitious materials design is control of the permeability, and hence durability, of the cement by control of the pore structure. That hydrogen can move between the gel and the capillary pore systems has important implications for developments of this kind. Until now, however, evidence for it has been lacking and it has remained a contested issue in the cement community.

\section{THEORY}

\section{Spectral density and $T_{1} / T_{2}$ ratio}

The observed spin lattice relaxation rate, $R_{1}=1 / T_{1}$, of spins (molecules) in a liquid filling a porous medium with a large surface-to-volume ratio, $S / V$, is dramatically enhanced compared to the bulk liquid rate by biphasic fast exchange of molecules between pore surface sites and the pore bulk liquid according to [1]

$$
\frac{1}{T_{1}(\omega)}=\frac{1}{T_{1, \text { bulk }}}+\frac{\varepsilon S}{V} \frac{1}{T_{1, \text { Surface }}(\omega)},
$$

where $\varepsilon$ is the thickness of the surface layer, assumed to be about the size of a single water molecule and $\omega / 2 \pi$ is the NMR frequency. The principal contribution to the overall rate comes from the surface term $1 / T_{1, \text { surface }}$ The problem therefore reduces to evaluating $T_{1, \text { surface }}$, a problem for which we have previously proposed a theory $[5,6]$. The theoretical model supposes that a large number of proton (spin $I$ ) bearing molecules such as water are diffusing at or close to a solid surface with a surface density $\sigma$ of fixed paramagnetic impurities $\left(\mathrm{Fe}^{3+}\right)$. The proton spin relaxation is primarily due to the modulation of the dipole-dipole interactions between

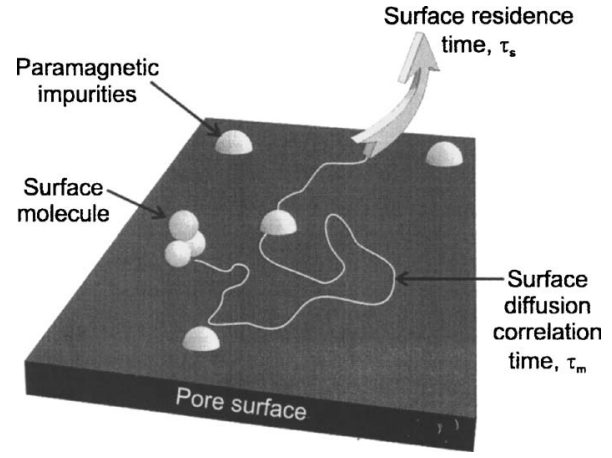

FIG. 1. Schematic of the two-dimensional diffusion of water molecules in the vicinity of $\mathrm{Fe}^{3+}$ paramagnetic ions on the solidliquid pore surface interface. The correlation time for molecular jumps is $\tau_{m}$; the surface residence time is $\tau_{s}$.

the $I$ and $S$ spins brought about by the translational diffusion of the $I$ spins past the fixed relaxation sinks, $S$. This is possible first because the gyromagnetic ratio of the electron, $\gamma_{S}$, is much larger than that of the proton, $\gamma_{I}\left(\gamma_{S}=658.21 \gamma_{I}\right)$, and second because there are numerous molecular reencounters between the $I$ and $S$ spins under conditions of a twodimensional random walk. There are far fewer reencounters in three dimensions. The model develops two correlation times associated with the probe molecule dynamics at the surface. One is the surface residence time, $\tau_{s}$, for probe molecules encountering the surface. It depends upon the existence of bonding sites for the probe molecule on the surface and is limited by molecular desorption. The other is the translational correlation time, $\tau_{m}$, associated with individual molecular jumps of adsorbed molecules which diffuse across the surface. A schematic visualization of the molecular surface motion is presented in Fig. 1.

These considerations lead, after some lengthy calculations fully detailed elsewhere $[5,6]$, to expressions for the spectral density functions for the motion $J_{L}^{0,1,2}(\omega)$ and thence to an expression for $T_{1, \text { surface }}(\omega)$. The theory predicts that the ${ }^{1} \mathrm{H}$ spin-lattice relaxation rate is linear in two parts when plotted as a function of the logarithm of the magnetic field strength and the slopes of these distinct linear regions should be in the ratio of $(10 / 3)$. The theoretical predictions follow closely the experimental frequency dependencies of $1 / T_{1}$ observed with field cycling proton relaxometry of various liquids confined in calibrated porous media [5-7]. These theoretical predictions have also been observed in cementitious materials during progressive hydration [16].

Using the notation of our previous work [5,6], we now take the general expression for the transverse and longitudinal relaxation rates [17],

$$
\begin{aligned}
\frac{1}{T_{1, \text { surface }}}= & \frac{2}{9} \gamma_{I}^{2} \gamma_{S}^{2} \hbar^{2} S(S+1)\left[J_{L}^{0}\left(\omega_{I}-\omega_{S}\right)+3 J_{L}^{1}\left(\omega_{I}\right)\right. \\
& \left.+6 J_{L}^{2}\left(\omega_{I}+\omega_{S}\right)\right]
\end{aligned}
$$




$$
\begin{aligned}
\frac{1}{T_{2, \text { surface }}}= & \frac{4}{9} \gamma_{I}^{2} \gamma_{S}^{2} \hbar^{2} S(S+1)\left(J_{L}^{0}(0)+\frac{1}{4} J_{L}^{0}\left(\omega_{I}-\omega_{S}\right)+\frac{3}{4} J_{L}^{1}\left(\omega_{I}\right)\right. \\
& \left.+\frac{3}{2} J_{L}^{1}\left(\omega_{S}\right)+\frac{3}{2} J_{L}^{2}\left(\omega_{I}+\omega_{S}\right)\right)
\end{aligned}
$$

[which can be simplified using the inequality $\omega_{S} \gg \omega_{I}\left(\omega_{S}\right.$ $\left.\left.=658.21 \omega_{I}\right)\right]$ and the previously calculated expression for the spectral density functions specific to the surface diffusion model at low frequency [5-7],

$$
J_{L}^{0,1,2}(\omega)=\frac{3}{40} \frac{\pi \sigma_{s}}{\delta^{4}} \tau_{m} \ln \left[\frac{1+\omega^{2} \tau_{m}^{2}}{\left(\frac{\tau_{m}^{2}}{\tau_{s}^{2}}\right)+\omega^{2} \tau_{m}^{2}}\right]
$$

to calculate an expression for the frequency dependence of the ratio $T_{2 \text {,surface }}(\omega) / T_{1 \text {, surface }}(\omega)$,

$$
\frac{T_{2, \text { surface }}(\omega)}{T_{1, \text { surface }}(\omega)}=2\left\{\frac{3 \ln \left(\frac{1+\omega_{I}^{2} \tau_{m}^{2}}{\left(\frac{\tau_{m}}{\tau_{S}}\right)^{2}+\omega_{I}^{2} \tau_{m}^{2}}\right)+7 \ln \left(\frac{1+\omega_{S}^{2} \tau_{m}^{2}}{\left(\frac{\tau_{m}}{\tau_{S}}\right)^{2}+\omega_{S}^{2} \tau_{m}^{2}}\right)}{4 \ln \left(\left(\frac{\tau_{S}}{\tau_{m}}\right)^{2}\right)+3 \ln \left(\frac{1+\omega_{I}^{2} \tau_{m}^{2}}{\left(\frac{\tau_{m}}{\tau_{S}}\right)^{2}+\omega_{I}^{2} \tau_{m}^{2}}\right)+13 \ln \left(\frac{1+\omega_{S}^{2} \tau_{m}^{2}}{\left(\frac{\tau_{m}}{\tau_{S}}\right)^{2}+\omega_{S}^{2} \tau_{m}^{2}}\right)}\right\}
$$

The most important feature of Eq. (5) is that the ratio $T_{2} / T_{1}$ depends only on the NMR frequency and the two correlation times, $\tau_{m}$ and $\tau_{s}$. In particular it is independent of all parameters such as the surface density of paramagnetic species $(\sigma)$ and the distance of minimum approach $(\delta)$ used in the derivation of Eq. (3).

Figure 2 shows a plot of the calculated dependence of the ratio of the two relaxation times as a function of the dimensionless variable $\omega_{I} \tau_{m}$ for three different values of the ratio $\tau_{m} / \tau_{s}$ from $10^{-2}$ to $10^{-4}$. It is worthwhile to note that the inverse ratio $\tau_{s} / \tau_{m}$ describes the number of molecular steps at the solid surface. The figure clarifies how the relaxation time ratio decreases dramatically from unity for twodimensional diffusion. In our experiments, $\omega_{I} / 2 \pi$ $=20 \times 10^{6} \mathrm{~Hz}$ and previous estimates suggest $\tau_{m} \approx 1.3$ $\times 10^{-9} \mathrm{~s}^{16}$, thus giving a value $\omega_{I} \tau_{m} \approx 0.16$. Hence, from Fig.

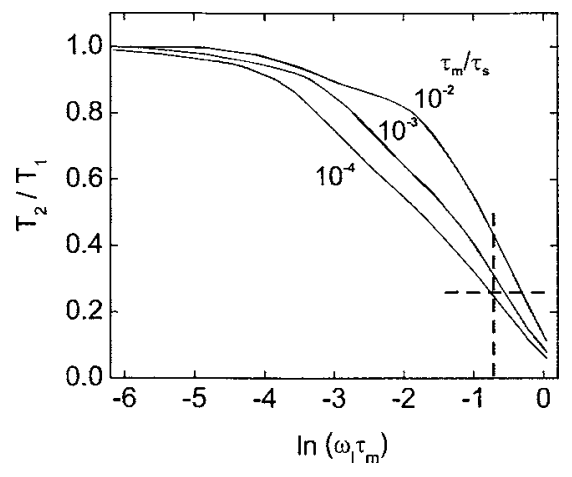

FIG. 2. The frequency dependence of the ratio $T_{2 \text {,surface }} / T_{1, \text { surface }}$ calculated from Eq. (5) for different values of the correlation time ratio, $\tau_{m} / \tau_{s}$, equal to $10^{-2}$ (upper curve), $10^{-3}$ (middle curve), and $10^{-4}$ (lower curve). The horizontal dotted line corresponds to the ratio $T_{1}=4 T_{2}$. It crosses the vertical line at $\omega_{I} \tau_{m} \approx 0.16$ (corresponding to the actual NMR frequency and $\tau_{m}=1.3 \mathrm{~ns}$ from Ref. [16]) for approximately $\tau_{m} / \tau_{s} \approx 10^{-4}$.
2 , we can predict a range from 0.25 to 0.50 for the expected value of the ratio $T_{2} / T_{1}$ as shown by the vertical dotted line.

\section{Cross relaxation}

In the absence of exchange, the time dependence of the magnetization data arising from a $T_{1}-T_{2}$ correlation experiment is described by

$$
\begin{aligned}
M\left(t_{1}, t_{2}\right)= & M_{0} \iint P\left(T_{1}, T_{2}\right)\left[1-2 \exp \left(\frac{-t_{1}}{T_{1}}\right)\right] \\
& \times \exp \left(\frac{-t_{2}}{T_{2}}\right) d T_{1} d T_{2},
\end{aligned}
$$

where $P\left(T_{1}, T_{2}\right)$ is the required $T_{1}-T_{2}$ distribution that is found by the two-dimensional inverse Laplace transform. Nuclear spin cross relaxation can occur as a result of chemical exchange, spin-diffusion and dipolar interactions. It modifies the observed relaxation rates. The general arguments have been well rehearsed in the literature. We reproduce the salient results following the notation due to Hill et al. [15]

During the $T_{1}$ encoding of a $T_{1}-T_{2}$ experiment, two coupled magnetization reservoirs, $M_{a}$ and $M_{b}$ relax according to

$$
\left[\begin{array}{l}
d M_{a} / d t \\
d M_{b} / d t
\end{array}\right]=\left[\begin{array}{cc}
-R_{a 1}-k_{a 1} & k_{b 1} \\
k_{a 1} & -R_{b 1}-k_{b 1}
\end{array}\right]\left[\begin{array}{c}
M_{a}-M_{a}^{0} \\
M_{b}-M_{b}^{0}
\end{array}\right],
$$

where it is understood that the magnetization is in the longitudinal direction. During the $T_{2}$ encoding the magnetization is turned into the transverse plane and relaxes according to 


$$
\left[\begin{array}{l}
d M_{a} / d t \\
d M_{b} / d t
\end{array}\right]=\left[\begin{array}{cc}
-R_{a 2}-k_{a 2} & k_{b 2} \\
k_{a 2} & -R_{b 2}-k_{b 2}
\end{array}\right]\left[\begin{array}{l}
M_{a} \\
M_{b}
\end{array}\right]
$$

where, for instance $R_{a 1}$ and $k_{a 1}$ are the $T_{1}$ and longitudinal cross relaxation rate for reservoir $a$ and $M_{a}^{0}$ is the equilibrium magnetization. The necessity for detailed balance requires that $M_{a}^{0} k_{a 1}=M_{b}^{0} k_{b 1}$ and $M_{a}^{0} k_{a 2}=M_{b}^{0} k_{b 2}$.

The solutions are

$$
\begin{aligned}
M_{a}\left(t_{1}, t_{2}\right)= & \frac{\left(s_{2}^{-}+R_{a 2}+k_{a 2}\right) m_{a}\left(t_{1}\right)-k_{b 2} m_{b}\left(t_{1}\right)}{s_{2}^{-}-s_{2}^{+}} \exp \left(s_{2}^{+} t_{2}\right) \\
& +\frac{\left(s_{2}^{+}+R_{a 2}+k_{a 2}\right) m_{a}\left(t_{1}\right)-k_{b 2} m_{b}\left(t_{1}\right)}{s_{2}^{+}-s_{2}^{-}} \exp \left(s_{2}^{-} t_{2}\right)
\end{aligned}
$$

and an equivalent, symmetric solution for $M_{b}$ given by interchanging $a$ and $b$ where

$$
\begin{aligned}
m_{a}\left(t_{1}\right)= & M_{a}^{0}-2 \frac{\left(s_{1}^{-}+R_{a 1}+k_{a 1}\right) M_{a}^{0}-k_{b 1} M_{b}^{0}}{s_{1}^{-}-s_{1}^{+}} \exp \left(s_{1}^{+} t_{1}\right) \\
& -2 \frac{\left(s_{1}^{+}+R_{a 1}+k_{a 1}\right) M_{a}^{0}-k_{b 1} M_{b}^{0}}{s_{1}^{+}-s_{1}^{-}} \exp \left(s_{1}^{-} t_{1}\right)
\end{aligned}
$$

and an equivalent expression for $m_{b}\left(t_{1}\right)$ are the magnetizations at the end of the first, start of the second, period of encoding and

$$
\begin{aligned}
s_{i}^{+/-}= & \frac{-1}{2}\left(R_{a i}+k_{a i}+R_{b i}+k_{b i}\right) \pm \frac{1}{2}\left(\left(R_{a i}+k_{a i}+R_{b i}+k_{b i}\right)^{2}\right. \\
& \left.-4\left(\left(R_{a i}+k_{a i}\right)\left(R_{b i}+k_{b i}\right)-k_{a i} k_{b i}\right)\right)^{1 / 2}
\end{aligned}
$$

are the effective relaxation rates. The total observed magnetization is the $\operatorname{sum} M_{a}\left(t_{1}, t_{2}\right)+M_{b}\left(t_{1}, t_{2}\right)$.

We have used Eqs. (9)-(11) to generate simulated data for a range of relaxation and cross coupling parameters. We have then inverted this data. In general, it leads to four peaks in the $2 \mathrm{D}$ relaxation correlation spectrum. These are expected at locations $\left(s_{1}^{+}, s_{2}^{+}\right),\left(s_{1}^{+}, s_{2}^{-}\right),\left(s_{1}^{-}, s_{2}^{+}\right)$, and $\left(s_{1}^{-}, s_{2}^{-}\right)$. In practice the diagonal peaks occur where expected although the offdiagonal peaks are slightly shifted. We attribute this to the inverse Laplace transform and note that the one-dimensional simulated data presented by Hills et al. shows a similar small discrepancy. Notwithstanding this limitation, under realistic conditions, one of the peaks is generally of negligible intensity, that at $\left(s_{1}^{-}, s_{2}^{+}\right)$. Under conditions of slow exchange $(R$ $\gg k)$, just two peaks are observed, at $\left(s_{1}^{+}, s_{2}^{+}\right)$and $\left(s_{1}^{-}, s_{2}^{-}\right)$ while under conditions of very rapid exchange $(k \gg R)$ a single peak is observed at a weighted average location.

In the case of a $T_{2}-T_{2}$ experiment, Eqs. (8)-(11) still apply except that $R_{a 2}=1 / T_{2 a}, R_{b 2}=1 / T_{2 b}, k_{a 2}$ and $k_{b 2}$ all act in both the first and second encoding periods so that

$$
\begin{aligned}
m_{a}\left(t_{1}\right)= & \frac{\left(s_{2}^{-}+R_{a 2}+k_{a 2}\right) M_{a}^{0}-k_{b 2} M_{b}^{0}}{s_{2}^{-}-s_{2}^{+}} \exp \left(s_{2}^{+} t_{1}\right) \\
& +\frac{\left(s_{2}^{+}+R_{a 2}+k_{a 2}\right) M_{a}^{0}-k_{b 2} M_{b}^{0}}{s_{2}^{+}-s_{2}^{-}} \exp \left(s_{2}^{-} t_{1}\right)
\end{aligned}
$$

(with a complementary equation for reservoir $b$ ). The inherent symmetry of the $T_{2}-T_{2}$ experiment ensures that the two off-diagonal exchange peaks have equal intensity. However, in a practical implementation of the experiment as described in the next section, a longitudinal storage period may be introduced between the two $T_{2}$ encoding intervals. This period, $\tau_{\text {delay }}$, serves to clearly demarcate between the two encoding intervals. During the storage, $T_{1}$ relaxation with exchange occurs. The introduction of $\tau_{\text {delay }}$ serves to break the symmetry of the experiment so that the two off-diagonal peaks are no longer necessarily equal. The relevant equations can be written down by extension of the foregoing analysis to three exchange periods leading to $M_{a}\left(t_{1}, \tau_{\text {delay }}, t_{2}\right)$ and so forth although this is not done here as the expressions quickly become unwieldy.

\section{MATERIALS AND METHODS}

Ketton white cement, obtained from Castle Cement, ${ }^{1}$ was used in all the samples. Small, carefully measured amounts were mixed in the water to cement ratio of 0.4 and cast in cylindrical moulds $10 \mathrm{~mm}$ in diameter and $20 \mathrm{~mm}$ deep. The samples were cured for $24 \mathrm{~h}$ in a high humidity environment before being removed from the moulds and placed in standard $10 \mathrm{~mm}$ NMR tubes. The first measurement (day 1) was made immediately. Thereafter, a small quantity of water was added so as to just cover the samples allowing them to cure underwater. They were stored at $20^{\circ} \mathrm{C}$. Prior to subsequent measurements the added water was removed, the outside of the sample gently dried with absorbent paper and the sample returned to a clean, dry NMR tube. The excess water was replaced after the measurement. Several repeats of the samples were prepared. A second series of samples with added silica fume was also made. These samples incorporated microsilica obtained from Elkem ${ }^{2}$ equal to $10 \%$ by mass of the cement.

The ESR spectrum of a white cement sample was acquired using a Bruker spectrometer operating at $9.6 \mathrm{GHz}$ in order to confirm the presence at low level of residual $\mathrm{Fe}^{3+}$ and $\mathrm{Mn}^{2+}$ paramagnetic ions. In order to quantify the ion content of the cement using the spectrum, a small single crystal of $\mathrm{CuSO}_{4}$ with a precisely measured mass was incorporated into the dry sample.

The basic 2D NMR relaxation correlation method is well documented in the literature $[10,11,13-15]$ although there are numerous variants upon it. The experiment consists of two parts during which the two dimensions are encoded. In our implementation of the $T_{1}-T_{2}$ correlation experiment, we use

\footnotetext{
${ }^{1}$ Castle Cement, Park Square, 3160 Solihull Parkway, Birmingham Business Park, Birmingham, B37 YN, U.K.

${ }^{2}$ Elkem Refactories, P. O. Box 8126, Vaagsbygd, N-4602 Kristainsand S., Norway.
} 
an inversion recovery followed by a Carr-Purcell-MeiboomGill (CPMG) pulse sequence defined by

$$
\begin{aligned}
& {\left[P_{180}-\alpha^{i} \tau_{1}-P_{90}-\beta^{0} \tau_{2}-\left(P_{180}-\beta^{j} \tau_{2}-(\text { echo })-\beta^{j+1} \tau_{2}\right)_{m}\right.} \\
& \left.\quad-\tau_{R D}\right]_{n} .
\end{aligned}
$$

Here, $P_{180}$ and $P_{90}$ are inversion and excitation radio frequency pulses; $\alpha^{i} \tau_{1}$ and $\left(\beta^{j}+\beta^{j+1}\right) \tau_{2}$ are the inversion recovery and echo sequence $180^{\circ}-180^{\circ}$ pulse gap intervals, respectively. The index $i$, the power to which the constant $\alpha$ is raised, is increased from 0 to $n-1$ over the $n$ repeats of the primary sequence enclosed in square brackets. Similarly, the index $j$ is increased from 0 to $m-1$ over the $m$ repeats of the echo sequence part of the sequence in round brackets. The inversion recovery interval and the echo pulse gap are therefore both increased logarithmically so as to span a very wide range of time intervals. The complex data sets are rotated to provide the $T_{1}$ inversion recovery in the "real" channel and the echo intensities from the CPMG measurements are recorded. In this way the experiment yields a two-dimensional $n \times m$ data set encoding $T_{1}$ in the first dimension and $T_{2}$ in the second. The time $\tau_{R D}$ is the repetition interval delay to allow full recovery of the magnetization between measurements.

In the experiments reported here, $n=50$ and $m=64$. The timing parameters are $\tau_{1}=100 \mu \mathrm{s}, \tau_{2}=34 \mu \mathrm{s}$, and $\tau_{R D}$ $=1.0 \mathrm{~s}$, with $\alpha=1.2068$ and $\beta=1.1351$. Accordingly, $t_{1 i}$ $=\alpha^{i} \tau_{1}, t_{2 j}=\sum_{k=0}^{j} 2 \beta^{k} \tau_{2}$. Hence the inversion recovery interval spans 0.1 to $1000 \mathrm{~ms}$ and the echo decay spans 0.068 to $1680 \mathrm{~ms}$. Each data set was averaged 128 times resulting in a total acquisition time of $5 \mathrm{~h}$. The measurements were made using a low-frequency $(20 \mathrm{MHz})$ bench-top Maran spectrometer manufactured by Resonance Instruments Ltd.

The $T_{2}-T_{2}$ correlation experiment pulse sequence has much in common with the $T_{1}-T_{2}$ sequence and is defined as

$$
\begin{aligned}
& {\left[P_{90}-\beta^{0} \tau_{2}-\left(P_{180}-\beta^{i}-\tau_{2}(\text { echo })-\beta^{i+1} \tau_{2}\right)_{i \max }-P_{90}\right.} \\
& \quad-\tau_{\text {delay }}-P_{-90}-\beta^{0} \tau_{2}-\left(P_{180}-\beta^{j} \tau_{2}(\text { echo })-\beta^{j+1} \tau_{2}\right)_{m} \\
& \left.\quad-\tau_{R D}\right]_{n} .
\end{aligned}
$$

The acquisition parameters used in both halves of the experiment were similar to those of the second half of the $T_{1}-T_{2}$ experiment with the addition that $i_{\max }$ is incremented from 0 to $n-1$ over the $n$ repeats of the sequence. The interval $\tau_{\text {delay }}$ describes a period of longitudinal storage of the magnetization which serves to clearly separate the first and second encoding period.

The Laplace inversion was performed using the method devised by Venkataramanan, Song, and Hurlimann [8] with software made available to us by Song. Others have made growing use of this method in recent years and there is now a sufficient body of literature experience that gives us considerable confidence in it. Nonetheless, we have performed inversions of simulated data with varying amounts of added white noise. Our experimental data has a signal-to-noise ratio at least equal to 400 to 1 . Analysis of simulated data of similar form to that recorded experimentally suggests that this is entirely adequate to have confidence in the features

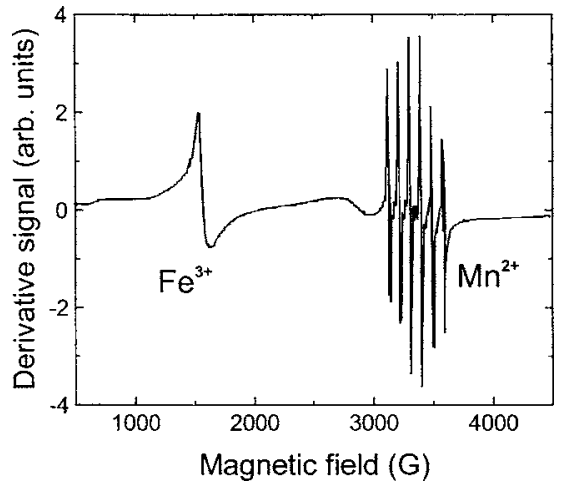

FIG. 3. The ESR spectrum of a white cement sample demonstrating the presence of a significant number of $\mathrm{Fe}^{3+}$ ions. A quantitative analysis of the respective contribution has been made both by an ESR calibration and chemical analysis (see text).

observed and conclusions reached even if, as already noted, some of the off-diagonal features are slightly shifted. In analyzing the intensity of the smaller features, white noise was repeatedly added to the real data at a similar level to the experimentally observed noise. The inversion was repeated each time and the average and standard deviation of the resultant parameters evaluated.

\section{RESULTS}

Figure 3 shows an ESR spectrum recorded from a white cement sample at room temperature. It confirms the presence not only of $\mathrm{Fe}^{3+}$ ions, but also a small quantity of $\mathrm{Mn}^{2+}$ ions. Using the $\mathrm{CuSO}_{4}$ crystal, we estimate $4.46 \times 10^{18} \mathrm{Fe}^{3+}$ ions/g and a negligible quantity of $\mathrm{Mn}^{2+}$. The surface area of the well resolved hyperfine structure of $\mathrm{Mn}^{2+}$ is in fact very low compared to the large surface area of the nonresolved ESR spectra of $\mathrm{Fe}^{3+}$. Moreover, these results have been confirmed by chemical analysis that gives 95 and $5 \%$ relative contributions for $\mathrm{Fe}^{3+}$ and $\mathrm{Mn}^{2+}$, respectively.

Figure 4 shows the raw NMR $T_{1}-T_{2}$ data set recorded from a sample of white cement that had been allowed to

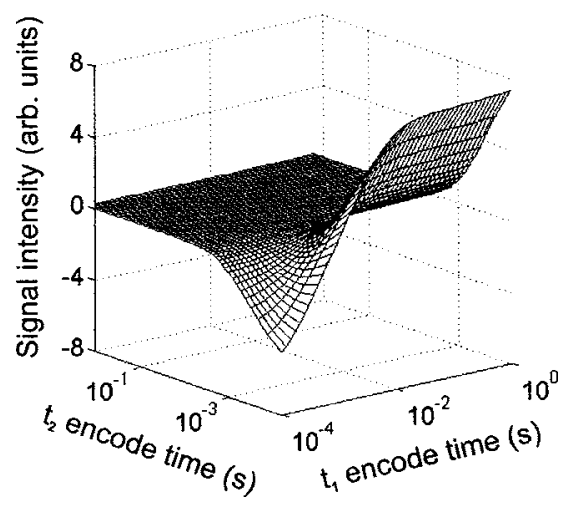

FIG. 4. Measured magnetization as a function of $t_{1}$ and $t_{2}$ measured from a sample of white cement after 4 days of curing. The inversion recovery [form of $\left.1-2 \exp \left(-t_{1}\right)\right]$ is clearly seen in the first dimension; the CPMG decay response $\left[\exp \left(-t_{2}\right)\right]$ in the second. The signal to noise ratio of the data exceeds 400 . 


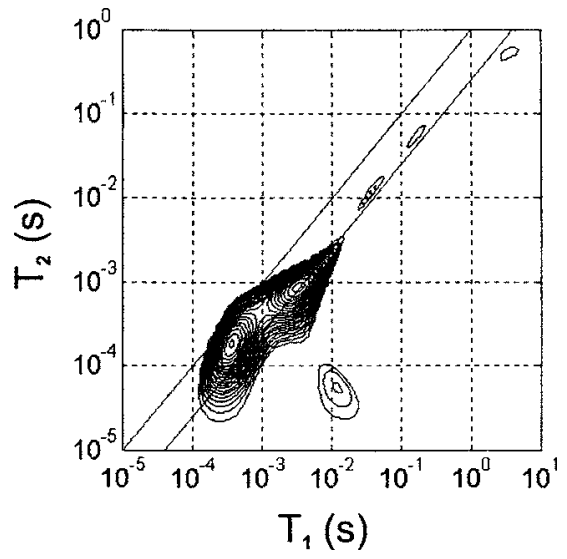

FIG. 5. The $T_{1}-T_{2}$ correlation spectrum of a white cement sample cured for 4 days. The spectrum is the $2 \mathrm{D}$ inverse Laplace transform of the data shown in Fig. 4. A series of discrete features lie along the line $T_{1}=4 T_{2}$ parallel to the diagonal. Both this line and the principal diagonal $\left(T_{1}=T_{2}\right)$ are shown as dotted lines. There is an off-diagonal feature at the position $T_{1}=10 \mathrm{~ms}$ and $T_{2}=50 \mu \mathrm{s}$. The intensity contours (arbitrary units) are equally spaced up from a zero base.

hydrate for 4 days. The manner in which the $T_{1}$ inversion recovery and the $T_{2}$ decay are encoded in the first and second dimension is readily apparent. The plot demonstrates the excellent signal to noise ratio-in excess of 400 to 1 - obtained in this experiment, a fact that greatly improves the stability of the inverse Laplace transform. Figure 5 shows the result of the 2D inverse Laplace transformation of this data set. The principal features are as follows: there is a series of five peaks aligned along a diagonal. The two of these with the shortest relaxation times, of the order of $200 \mu \mathrm{s}$ and $2 \mathrm{~ms}$, respectively, account for most of the intensity in the distributions; the other three are at longer relaxation times and are considerably smaller. All the peaks, but particularly the smaller ones, are well separated and discrete with approximately 1 order of magnitude in relaxation time between them. The second observation is that the diagonal features are, in fact, shifted slightly down from the principal diagonal, $T_{1}=T_{2}$, aligned instead along $T_{1}=4 T_{2}$. There is a single off-diagonal feature. Its position is close to that expected of a cross-peak connecting the two principal diagonal features. It is of low intensity but is highly reproducible having been seen in many experiments. Finally in regard to this data, we note that of course there is no symmetric feature above the diagonal.

Figure 6 contains a time series of plots that show the evolution of the $T_{1}-T_{2}$ correlations over the first 14 days of hydration for both the white cement paste (the same series from which Fig. 5 is extracted) and the cement paste with added silica fume. The plots shown are for the samples after $1,2,7$, and 14 days of curing. Three observations are made. First, the diagonal peaks in the silica fume paste are not as discrete as they are in the white cement paste. By day 14, they form an almost continuous ridge across the plot. Second, the off-diagonal peak drifts noticeably toward longer $T_{1}$ times as the reaction proceeds. The vertical dotted lines across the plots are at $T_{1}=10 \mathrm{~ms}$ and provide a good guide
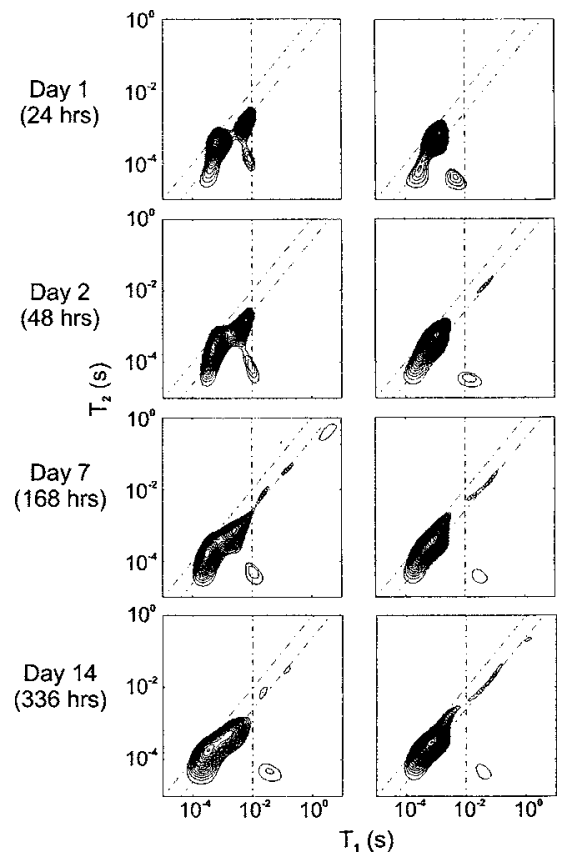

FIG. 6. Left, a time series of plots similar to those in Fig. 4 for the white cement sample. The plots shown are recorded after 1, 2, 7, and 14 days of curing. Right, a similar time series for the white cement sample with added silica fume. The plots can be directly compared as they are all plotted on the same vertical scale. The dotted lines are a guide to the eye at $T_{1}=10 \mathrm{~ms}$ designed to highlight the evolution of the shift of the off-diagonal peak. The intensity contours (arbitrary units) are equally spaced up from a zero base.

against which this phenomenon can be seen. Third, and in so much as this can be gauged from the plots, the reaction proceeds more quickly in the silica fume paste compared to the white cement paste. For instance, the shift in the off-diagonal feature already noted is not fully apparent until day 14 in the white cement. However, in the silica fume paste, the shift is already starting to occur on day 2. Equally, the smaller diagonal features appear for the first time at day 4 in the white cement paste (Fig. 5) but are already apparent on day 2 in the silica fume paste.

The final correlations discussed here are shown in Figs. 7 and 8 . These are the results of $T_{2}-T_{2}$ correlation experiments with delay interval of $\tau_{\text {delay }}=2$ and $10 \mathrm{~ms}$ between the two halves of the experiment, respectively. The former (Fig. 7) is recorded from a white cement paste after just one day of hydration, the latter (Fig. 8) after 4 days. The most important features of Fig. 7 are the two off-diagonal features placed symmetrically about the true diagonal, $T_{2}^{1}=T_{2}^{2}$, together with the two associated diagonal features completing a "square" in the lower left of the figure. This is consistent with expectation for a $T_{2}-T_{2}$ correlation experiment with a short (ideally zero) longitudinal storage interval. The peak at longest $T_{2}$ in this plot, in the upper right, is surprisingly displaced above the diagonal. One possible reason for this is diffusion of water through magnetic susceptibility field gradients as discussed in the next section. Turning to Fig. 8, three diagonal features are seen. Continuing a trend begun in Fig. 7, the upper two are now strongest, the lower one relatively weak. 


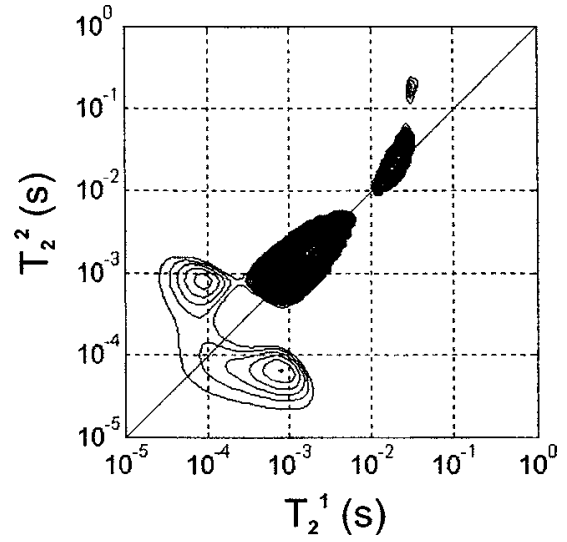

FIG. 7. The $T_{2}-T_{2}$ correlation spectrum of the white cement sample cured for 1 day recorded with a longitudinal storage interval of $2 \mathrm{~ms}$. The spectrum is very similar to the $T_{1}-T_{2}$ spectra except that the primary features now all lie along the principal diagonal: $T_{2}^{1}=T_{2}^{2}$. Also, off-diagonal peaks are now seen symmetrically placed both above and below the diagonal so forming a "square" in the lower left of the plot. This is the primary evidence presented that the off-diagonal feature in the previous plots is due to exchange of magnetisation between different environments rather than, e.g., due to chemically bound water. The fact that the peak at longest $T_{2}$ in the top right is above the diagonal is likely an artefact, perhaps associated with diffusion of water through susceptibility gradients. The intensity contours (arbitrary units) are equally spaced up from a zero base.

This is due to the interval $\tau_{\text {delay }}$, which filters short $T_{1}$ components. The effect is accentuated because the sample is older, the average $T_{1}$ is decreasing. Nonetheless, all three are again well centered on the true diagonal $T_{2}^{1}=T_{2}^{2}$ rather than on a line parallel to the diagonal. A small but reproducible single off-diagonal peak remains at the position of a lower cross peak for the lower two diagonal peaks. There is no symmetric peak on the upper side of the diagonal, presum-

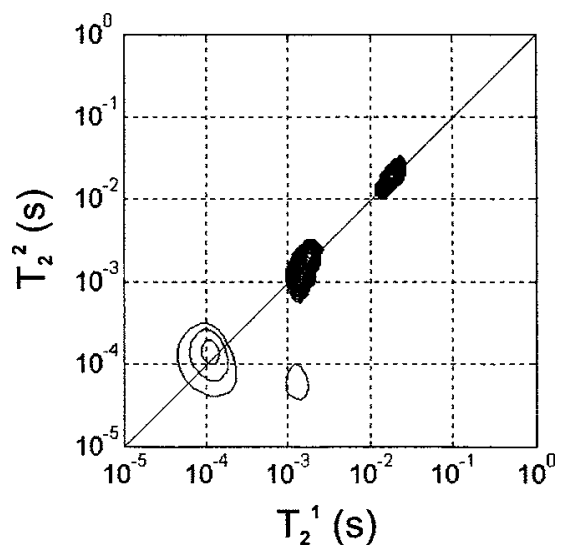

FIG. 8. The $T_{2}-T_{2}$ correlation spectrum of the white cement sample cured for 4 days recorded with a longitudinal storage interval of $10 \mathrm{~ms}$. Compared to Fig. 7, the relative intensities of the peaks change due to the longer $\tau_{\text {delay }}$ which serves to filter short $T_{1}$ components (so that intensity is lost in the lower left) and to break the symmetry of the experiment as regards the formation of offdiagonal exchange peaks (only one is seen). The intensity contours (arbitrary units) are equally spaced up from a zero base. ably due to the storage interval breaking the symmetry of the experiment.

\section{DISCUSSION}

Each of the discrete features along the diagonal of plots such as those in Figs. 5-8 corresponds to water in a different pore size environment. The existence of gel pores on a scale of some $20 \AA$ is well known in cement. The relaxation of water within these is assigned to the primary feature with the lowest relaxation time. The $T_{1}$ of circa $200 \mu \mathrm{s}$ is consistent with previous (1D) measurements of $T_{1}$ in similar cement pastes at similar NMR frequencies [18]. All the other features (with the exception of that at the longest $T_{1}$ relaxation time on the order of $3 \mathrm{~s}$ which is seen in some measurements), are attributed to the relaxation of water in capillary pores of increasing size as one moves up the diagonal. Clearly, the largest fraction is associated with a $T_{1}$ some 10 times longer than that of the relaxation time associated with gel water. This, too, is consistent with previous analyses. Multiple discrete modes have been observed previously in one-dimensional relaxation experiments, this analysis makes them particularly clear. The small component with the very long relaxation time is attributed to a layer of residual bulk water (used to prevent water loss during storage) remaining on the sample surface after they have been dabbed dry. Interestingly, it lies on $T_{1}=4 T_{2}$.

At first sight, the fact that multiple discrete capillary pore size modes can be distinguished is somewhat surprising as most conventional analyses, e.g., by mercury intrusion porosimetry, suggest a continuous distribution in cement. However, detailed atomic force microscopy studies of hydrating cements do offer supportive evidence of structure at different discrete length scales [19] as does an alternative NMR based method of measuring pore size distributions, NMR cryoporometry [20]. According to the Gibbs-Thompson equation, the melting point of liquids confined to small pores is depressed compared to that of the bulk liquid by an amount inversely proportional to the pore size. Milia et al. also showed that the freezing point of water in cements is dependent on the pore size [21]. Valckenborg et al. [22] measured the pore size distributions in mortar samples via both cryoporometry and relaxometry. The measurements indicated three nominal pore sizes, although the shape of the distributions was different in the two techniques. It now seems clear that NMR methods that directly probe pore water, albeit in very different ways, repeatedly report a distribution of discrete pore sizes in hydrating cement whereas other more conventional analyses report a continuous distribution. These other techniques-not least mercury porosimetry-are generally more invasive than NMR and may be destroying the finer details of the structure in the course of the analysis. Equally, most of them are sensitive to the size of the pore throat rather than the pore itself and these dimensions are unlikely to be the same.

All the experimental evidence in this study suggests that $T_{1} \approx 4 T_{2}$ in the cement pastes studied. Figure 2 shows a short horizontal dotted line at $T_{1}=4 T_{2}$. It is seen to cross each of the curves in a narrow range of values of the dimensionless 
variable $\omega_{I} \tau_{m}: 0.1<\omega_{I} \tau_{m}<1$. The short vertical line is for $\omega_{I} \tau_{m}=0.16$, corresponding to the actual NMR frequency and a value of $\tau_{m}$ of $1.3 \mathrm{~ns}$ taken from Ref. [16]. These lines intersect the lower curve which leads us to the conclusion that $\tau_{m} / \tau_{s}=10^{-4}$. This observation is offered as strong supportive evidence for the validity of the surface diffusion model of relaxation. We note that, reviewing the literature, other earlier results can now be explained. In particular, Hurlimann and Venkataramanan [10] reported 2D $T_{1}-T_{2}$ measurements for Indiana limestone and showed a long diagonal feature spanning more than 2 orders of magnitude in relaxation time which was interpreted as a broad and continuous distribution of pore sizes. A detailed examination shows that this distribution is shifted down from the $T_{1}=T_{2}$ line to nearer $T_{1}=2 T_{2}$. Such a result is explicable in terms of surface diffusion dominated relaxation. Also, Korb et al. [23] previously made measurements of the (1D) relaxation times of simple liquids confined to model microporous systems. $T_{1} / T_{2}$ ratios as great as a factor of 10 were observed.

The existence of an off-diagonal feature in $T_{1}-T_{2}$ correlations could be attributed to hydrogen in a solidlike environment, for instance hydrogen chemically combined into the cement, especially as $T_{1} \gg T_{2}$ for the experimentally observed feature. We discount this possibility for two reasons. First, the observed $T_{2}$ of the off-diagonal peak is substantially longer, by almost an order of magnitude, than that normally reported for bound hydrogen in cement. Second, and more fundamentally, we see the off-diagonal peaks also in the $T_{2}-T_{2}$ correlations. Bound hydrogen along with all the rest would give rise to a diagonal feature in this experiment in the absence of any exchange mechanism. Rather, we attribute the off-diagonal peaks to a chemical exchange of hydrogen (water?) between pores of different sizes. An offdiagonal peak corresponds to hydrogen magnetization in the pore environment associated with the $T_{1}$ (or $T_{2}$ ) of the vertically connected diagonal feature exchanging with hydrogen magnetization in an environment associated with the $T_{2}$ of the horizontally connected diagonal feature. Such a situation arises if the proton moves between two pore sizes on the timescale of the measurement: chemical exchange. Since the off-diagonal features link diagonal peaks corresponding to capillary and gel pore water, we infer transfer of hydrogen (water?) between the capillaries and the gel. We believe that this is the first time that evidence for such a capillary-gel interaction has been presented. The fact that corresponding peaks indicative of transfer the other way (gel to capillary) are not seen the other side of the diagonal in the $T_{1}-T_{2}$ experiments is reassuring, but is not evidence that it does not occur. Such a peak would require $T_{1}<T_{2}$ which is not physically possible. The magnetization coherence required for a long $T_{2}$ cannot be maintained once magnetization has returned to the longitudinal direction and once it has already dephased in a small pore environment. Additionally, since one might expect transform artefacts to be symmetrically dispersed around the diagonal, the absence of a cross peak on the upper diagonal side further suggests that the observed peaks are genuine.

Figure 9 shows the intensity (integrated peak volume) ratio of the off-diagonal feature compared to the principal two diagonal features. In the case of the white cement there is no

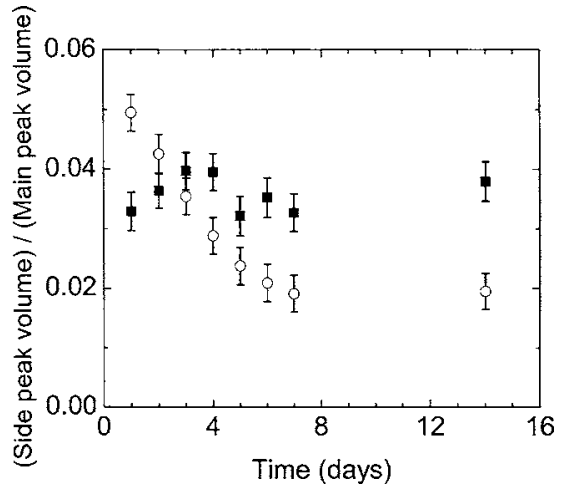

FIG. 9. The ratio of the exchange peak intensity to the total diagonal intensity as measured from $T_{1}-T_{2}$ correlation spectra such as those in Fig. 6 as a function of sample curing time for both a white cement sample (solid squares) and a white cement sample with added silica fume (open circles).

clear trend in this ratio, it remains largely constant throughout. In the case of the silica fume paste it decreases systematically during the first few days of curing. This likely reflects detailed differences in the evolving microstructure and perhaps that there is less water available for exchange in the capillary pore reservoir in the silica fume paste.

The experiments using white cement with added silica fume show an earlier onset of features than those with pure white cement. The silica fume vastly increases the available surface area on which the cement hydration reactions can occur and consumes the lime produced in those same reactions that normally inhibits the production of the CSH gel. This results in an acceleration of the curing. The NMR evidence from this study is that it disrupts the pore size distribution of the developing larger pores so as to create a more continuous distribution. It also appears to reduce the exchange of water between gel and capillary pores appreciably as curing proceeds. This could well be a consequence of the modified pore structure.

A careful study of the principal diagonal features shows an evolution of shape as the sample cure proceeds. Presumably changing shape reflects a changing pore structure environment and evolving $\tau_{m}$, although this has not yet been fully interpreted. Features to be explained include the fact that the diagonal gel pore peak is slightly angled across the $T_{1}=4 T_{2}$ diagonal and that it has a tendency to fall slightly above this diagonal, perhaps at $T_{1}=2 T_{2}$. One possibility is that this is a direct result of the more regular, better defined two dimensional lamellar structure of the gel pores compared to the more irregular capillary pores.

Our analysis so far has ignored the effects of attenuation of magnetisation coherence during $T_{2}$ encoding by diffusion of water through the internal magnetic field gradients of the porous structure. Such gradients necessarily exist due to the different magnetic susceptibilities of the matrix and pore water. For a CPMG experiment with constant pulse gap $\tau$ applied in a constant magnetic field gradient, the effective $T_{2}$ is given by [24] 


$$
\frac{1}{T_{2}^{*}}=\frac{1}{T_{2}}+\frac{1}{3} \gamma^{2} G^{2} \tau^{2} D
$$

which in the limit of small $\tau$ or $G$ tends to the "true" value. Mitigating against this being a serious problem more widely in our experiments is the fact that we use a relatively low NMR frequency and hence applied magnetic field. Susceptibility gradients scale with field strength and the attenuation depends on the square of the gradient strength. Also, we use white cement: the paramagnetic impurity density is relatively low limiting the susceptibility difference across the pore wall interface. Elsewhere [10], in studies of rock, others have used very many echoes with a small constant pulse gap. However, the $\tau$ used was not that small and would not have accessed the gel water as we have done with a minimum $\tau$ of $30 \mu \mathrm{s}$. Also, there are problems with recording a very large number of echoes. First, the magnetization can spin-lock along the applied pulse field direction so that the measured $T_{2}$ is closer to the spin lattice relaxation time in the rotating frame Second the applied radio frequency energy can heat the sample and hence accelerate curing in a long experiment. Third, the volume of data recorded can become exceedingly large. For all of these reasons we chose to systematically increase the pulse gap throughout the echo train. Exactly how this impacts on the data is unclear although it may have a bearing on some of the finer details of the experimental results. For instance, one manifestation may be the position of the peak in the upper right of Fig. 7, the peak is noticeably displaced from the diagonal to a position above the diagonal. The fact that this is a $T_{2}-T_{2}$ experiment on a young sample may be relevant as diffusion selectively affects $T_{2}$ and the sample is less mature and therefore has more mobile water.

\section{CONCLUSION}

We have made the first $T_{1}-T_{2}$ and $T_{2}-T_{2} 2 \mathrm{D}$ nuclear spin relaxation correlation measurements of cement pastes. These experiments have provided the first clear evidence for chemical exchange of water protons between gel and capillary pores and have also provided further evidence in support of the two-dimensional surface diffusion model of spin relaxation in cements. Differences between white cement paste and white cement paste with added silica fume have been observed which reflect current understanding of the different microstructures.

\section{ACKNOWLEDGMENTS}

The authors thank Y.-Q. Song of Schlumberger-Doll Research for the use of a copy of his 2D fast Laplace inversion (FLI) software. One of the authors (L.M.) thanks the Nanocem Consortium for financial support. One of the authors (J.M.) thanks The Royal Society for financial support. The authors thank M. Mulheron of The University of Surrey for useful discussions and advice on making the samples.
[1] K. R. Brownstein and C. E. Tarr Phys. Rev. A 19, 2446 (1979).

[2] L. J. Schreiner, J. C. MacTavish, L. Miljkovic, M. M. Pintar, R. Blinc, G. Lahajnar, D. D. Lasic, and L. W. Reevesm, J. Am. Ceram. Soc. 68, 10 (1985).

[3] J. Greener, H. Peemoeller, C. Choi, R. Holly, E. J. Reardon, C. M. Hansson, and M. M. Pintar, J. Am. Ceram. Soc. 83, 623 (2000).

[4] D. D. Lasic, J. M. Corbett, J. Jian, J. C. MacTavish, M. M. Pintar, R. Blinc, and G. Lahajnar, Cem. Concr. Res. 18, 649 (1988).

[5] J.-P. Korb, M. Whaley-Hodges, and R. G. Bryant, Phys. Rev. E 56, 1934 (1997).

[6] S. Godefroy, J.-P. Korb, M. Fleury, and R. G. Bryant, Phys. Rev. E (to be published).

[7] J.-P. Korb, M. W. Hodges, Th. Gobron, and R. G. Bryant, Phys. Rev. E 60, 3097 (1999).

[8] L. Venkataramanan, Y. Q. Song, and M. D. Hurlimann, IEEE Trans. Signal Process. 50, 1017 (2002).

[9] Y. Q. Song, L. Venkataramanan, and M. D. Hurlimann, J. Magn. Reson. 154, 261 (2002).

[10] M. D. Hurlimann and L. Venkataramanan, J. Magn. Reson. 157, 31 (2002).

[11] B. Sun and K.-J. Dunn, J. Magn. Reson. 172, 152 (2005).

[12] J. G. Seland, K. E. Washburn, H. W. Anthonsen, and J. Krane,
Phys. Rev. E 70, 051305 (2004).

[13] P. T. Callaghan, S. Godefroy, and B. N. Ryland, Magn. Reson. Imaging 21, 243 (2003).

[14] P. T. Callaghan and I. Furo, J. Chem. Phys. 120, 4032 (2004).

[15] B. Hills, S. Benamira, N. Marigheto, and K. Wright, Appl. Magn. Reson. 26, 543 (2004).

[16] F. Barberon, J.-P. Korb, D. Petit, V. Morin, and E. Bermejo, Phys. Rev. Lett. 90, 116103 (2003).

[17] A. Abragam, Principles of Nuclear Magnetism (Oxford University Press, Oxford, 1961).

[18] E. Laganas, G. Papavassiliou, M. Fardis, A. Leventis, F. Milia, E. Chanioootakis, and C. Meletiou, J. Appl. Phys. 77, 3343 (1995).

[19] S. Gauffinet, E. Finot, E. Lesniewska, and A. Nonat, C. R. Acad. Sci., Ser. I: Math. 327, 231 (1998).

[20] J. H. Strange, M. Rahman, and E. G. Smith, Phys. Rev. Lett. 71, 3589 (1993).

[21] F. Milia, M. Fardis, G. A. Papavassiliou, and A. Leventis, Magn. Reson. Imaging 16, 677 (1998).

[22] R. M. E. Valckenborg, L. Pel, and K. Kopinga, J. Phys. D 35, 249 (2002).

[23] J.-P. Korb, A. Delville, S. Xu, G. Demeulenaere, P. Costa, and J. Jonas, J. Chem. Phys. 101, 7074 (1994).

[24] H. Y. Carr and E. M. Purcell, Phys. Rev. 94, 630 (1954). 